\title{
Effects of the particle-number projection on the isovector pairing energy
}

\author{
N.H. Allal ${ }^{1,2, a}$, M. Fellah ${ }^{1,2}$, M.R. Oudih ${ }^{1}$, and N. Benhamouda ${ }^{1}$ \\ 1 Laboratoire de Physique Theorique, Faculte de Physique, USTHB, BP 32 El-Alia, 16111 Bab-Ezzouar, Alger, Algeria \\ 2 Centre de Recherche Nucleaire d'Alger, COMENA, BP 399 Alger-Gare, Alger, Algeria
}

Received: 7 June 2005 /

Published online: 13 March 2006 - C Società Italiana di Fisica / Springer-Verlag 2006

\begin{abstract}
The usual neutron-proton BCS wave function is simultaneously projected on both the good neutron and proton numbers using a discrete projection operator. The projected energy of the system is deduced as a limit of rapidly convergent sequence. It is numerically studied for the $N=Z$ nuclei of which "experimental" pairing gaps may be deduced from the experimental odd-even mass differences. It then appears that the particle-number fluctuation effect is even more important than in the case of pairing between like-particles.
\end{abstract}

PACS. 21.60.-n Nuclear structure models and methods - 21.30.Fe Forces in hadronic systems and effective interactions

\section{Introduction}

Renewed interest in the study of the neutron-proton $(\mathrm{np})$ pairing correlations occurred recently (cf., e.g., $[1,2,3,4,5$, $6,7,8,9,10,11,12,13,14,15,16,17,18,19,20,21])$ due to the development of the radioactive beam facilities that made possible the experimental study of medium mass nuclei such as $N \simeq Z$. The study of these proton-rich nuclei is not only interesting from the nuclear structure point of view, but it is also important in the astrophysical context. Indeed, it is believed that this kind of nuclei are synthesized in the rapid-proton capture process (rp-process) under astrophysical conditions [22,23]. Moreover, most of these nuclei are not accessible in experiments and therefore many nuclear astrophysics calculations crucially depend on accurate theoretical prediction for the nuclear properties. In $N \simeq Z$ nuclei, the neutron and proton Fermi levels are close to each other and therefore the np pairing correlations are expected to play a significant role in their structure.

On the other hand, one of the most exciting subjects of the modern nuclear physics is the double-beta decay, because one expects an answer to the question whether the neutrino is a Majorana or a Dirac particle (cf., e.g., [24, $25,26,27])$. Indeed, neutrinoless double-beta decay proceeds only when neutrinos are massive Majorana particles, hence its observation would resolve the question [28]. It has been shown that the inclusion of the np pairing

\footnotetext{
a e-mail: allaln@yahoo.com
}

influences the neutrinoless double-beta decay rates significantly [9].

Most often, pairing is treated within a generalized BCS treatment $[1,2,3,4,5,6,7,8]$. However, the shortcomings of this approach are well known: the most important of them is the number symmetry breaking that may imply serious errors in the evaluation of several physical observables. The usual techniques used in order to remedy this shortcoming are those already used for the study of pairing correlations between likeparticles, i.e. the Quasiparticle Random Phase Approximation (QRPA) (cf., e.g., $[9,10,11,12,13,14,15,16,17])$, the Lipkin-Nogami (LN) method [18] or the Generator Coordinate Method (GCM) [19]. However, in these methods, the particle-number symmetry is only approximately restored. Recently, an exact particle-number projection method in the isovector case has been proposed [29]. It is based on a discrete form of the projection operator that allows one to derive an explicit form of the projected wavefunction. The method was applied within the Richardson schematic model [30]. The aim of the present work is to study numerically the particle-number fluctuations effects on the energy in a realistic case, i.e. for the $N=Z$ nuclei of which the "experimental" pairing gaps $\Delta_{p}, \Delta_{n}$ and $\Delta_{n p}$ may be deduced from the experimental odd-even mass differences [3]. The single-particle energies used are those of a Woods-Saxon deformed mean field. The structure of this paper is as follows: the BCS theory is briefly recalled in sect. 2. The projection method is presented in sect. 3 . The numerical results are given and discussed in sect. 4 . 


\section{BCS theory}

In the second quantization and isotopic spin formalism, a system of mass $A$ constituted by $N$ neutrons and $Z$ protons is described by the Hamiltonian [1]

$$
\hat{H}=\sum_{\nu t} \varepsilon_{\nu t} a_{\nu t}^{+} a_{\nu t}-\frac{1}{4} \sum_{\nu \mu>0, t_{1} t_{2}} G_{t_{1} t_{2}} a_{\nu t_{1}}^{+} a_{\tilde{\nu} t_{2}}^{+} a_{\tilde{\mu} t_{2}} a_{\mu t_{1}}
$$

where:

- $t$ is the subscript corresponding to the isotopic spin component $(t=n, p)$.

$-a_{\nu t}^{+}$and $a_{\nu t}$ respectively represent the creation and annihilation operators of the particle in the state $|\nu t\rangle$, of energy $\varepsilon_{\nu t}$; and $a_{\tilde{\nu} t}^{+}$and $a_{\tilde{\nu} t}$ those of its time-reverse $|\tilde{\nu} t\rangle$, that has the same energy.

- $G_{t_{1} t_{2}}$ characterizes the pairing strength and is supposed independent of the levels.

The neutrons and protons are supposed to occupy the same energy levels. The standard procedure is to use the generalized Bogoliubov-Valatin transformation approach $[1,2,3,8,31]$ where the quasi-particle operators are given by

$$
\left(\begin{array}{c}
\alpha_{\nu 1}^{+} \\
\alpha_{\nu 2}^{+} \\
\alpha_{\tilde{\nu} 1} \\
\alpha_{\tilde{\nu} 2}
\end{array}\right)=\left(\begin{array}{cccc}
U_{\nu 1 p} & U_{\nu 1 n} & V_{\nu 1 p} & V_{\nu 1 n} \\
U_{\nu 2 p} & U_{\nu 2 n} & V_{\nu 2 p} & V_{\nu 2 n} \\
-V_{\nu 1 p} & -V_{\nu 1 n} & U_{\nu 1 p} & U_{\nu 1 n} \\
-V_{\nu 2 p} & -V_{\nu 2 n} & U_{\nu 2 p} & U_{\nu 2 n}
\end{array}\right)\left(\begin{array}{c}
a_{\nu p}^{+} \\
a_{\nu n}^{+} \\
a_{\tilde{\nu} p} \\
a_{\tilde{\nu} n}
\end{array}\right)
$$

The indices 1, 2 reveal the existence of two kinds of quasiparticles.

The BCS state $|\Psi\rangle$ is obtained by eliminating all the quasiparticles in the actual vacuum, i.e.

$$
|\Psi\rangle \propto \prod_{\nu>0} \alpha_{\nu 1} \alpha_{\tilde{\nu} 1} \alpha_{\nu 2} \alpha_{\tilde{\nu} 2}|0\rangle
$$

Using the transformation (2), one obtains, after normalization,

$$
|\Psi\rangle=\prod_{\nu>0}\left|\Psi_{\nu}\right\rangle
$$

with

$$
\begin{aligned}
\left|\Psi_{\nu}\right\rangle= & {\left[B_{1}^{\nu} a_{\tilde{\nu} p}^{+} a_{\nu p}^{+} a_{\tilde{\nu} n}^{+} a_{\nu n}^{+}+B_{2}^{\nu} a_{\tilde{\nu} p}^{+} a_{\nu p}^{+}+B_{3}^{\nu} a_{\tilde{\nu} n}^{+} a_{\nu n}^{+}\right.} \\
& \left.+B_{4}^{\nu}\left(a_{\tilde{\nu} p}^{+} a_{\nu n}^{+}+a_{\tilde{\nu} n}^{+} a_{\nu p}^{+}\right)+B_{5}^{\nu}\right]|0\rangle
\end{aligned}
$$

and where the $B_{i}^{\nu}$ factors depend on the $U_{\nu \tau t}$ and $V_{\nu \tau t}$ $(t=n, p ; \tau=1,2)$ coefficients.

The pairing gap parameters are defined by

$$
\Delta_{t t^{\prime}}=\frac{G_{t t^{\prime}}}{2} \sum_{\nu>0}\left\langle\Psi\left|a_{\tilde{\nu} t} a_{\nu t^{\prime}}\right| \Psi\right\rangle, \quad t, t^{\prime}=n, p .
$$

The particle-number fluctuation will be measured by the quantity

$$
\Delta N_{B C S}=\frac{1}{A} \sqrt{\left\langle\Psi\left|\hat{N}^{2}\right| \Psi\right\rangle-\langle\Psi|\hat{N}| \Psi\rangle^{2}}
$$

where $\hat{N}$ is the particle-number operator.

The BCS energy is defined by $E_{B C S}^{n p}=\langle\Psi|\hat{H}| \Psi\rangle$, that is

$$
E_{B C S}^{n p}=\sum_{\nu>0} E_{\nu}+\sum_{\nu \neq \mu} E_{\nu \mu},
$$

where $E_{\nu}$ and $E_{\nu \mu}$ are evaluated in the particle-number representation:

$$
\begin{aligned}
E_{\nu}= & \left(B_{1}^{\nu}\right)^{2}\left[2 \varepsilon_{\nu n}+2 \varepsilon_{\nu p}-\frac{G_{n n}}{4}-\frac{G_{p p}}{4}-\frac{G_{n p}}{2}\right] \\
& +\left(B_{2}^{\nu}\right)^{2}\left[2 \varepsilon_{\nu p}-\frac{G_{p p}}{4}\right]+\left(B_{3}^{\nu}\right)^{2}\left[2 \varepsilon_{\nu n}-\frac{G_{n n}}{4}\right] \\
& +2\left(B_{4}^{\nu}\right)^{2}\left[\varepsilon_{\nu n}+\varepsilon_{\nu p}-\frac{G_{n p}}{4}\right]
\end{aligned}
$$

and

$$
\begin{aligned}
E_{\nu \mu}= & -\frac{G_{n n}}{4}\left(B_{1}^{\nu} B_{2}^{\nu}+B_{3}^{\nu} B_{5}^{\nu}\right)\left(B_{1}^{\mu} B_{2}^{\mu}+B_{3}^{\mu} B_{5}^{\mu}\right) \\
& -\frac{G_{p p}}{4}\left(B_{1}^{\nu} B_{3}^{\nu}+B_{2}^{\nu} B_{5}^{\nu}\right)\left(B_{1}^{\mu} B_{3}^{\mu}+B_{2}^{\mu} B_{5}^{\mu}\right) \\
& -\frac{G_{n p}}{2} B_{4}^{\nu}\left(B_{1}^{\nu}-B_{5}^{\nu}\right) B_{4}^{\mu}\left(B_{1}^{\mu}-B_{5}^{\mu}\right) .
\end{aligned}
$$

\section{Projection}

\subsection{Projected wave function}

In the case of pairing between identical particles, the projector that allows one to obtain the state of an even system with $2 P_{t}$ particles (where $P_{t}$ refers to the neutron or proton pair number), from the BCS vacuum, is given by [32]

$\hat{\wp}_{t}=\frac{1}{2(n+1)}\left\{\sum_{k=0}^{n+1} \xi_{k} z_{k}^{-P_{t}} \prod_{\nu}\left[1+\left(\sqrt{z_{k}}-1\right) a_{\nu t}^{+} a_{\nu t}\right]+\right.$ c.c. $\}$,

where

$$
\xi_{k}= \begin{cases}1 / 2, & \text { if } k=0 \text { or } k=n+1 \\ 1, & \text { if } 1 \leq k \leq n\end{cases}
$$

$z_{k}=\exp \left(i \frac{k \pi}{n+1}\right), n$ is a non-zero integer and c.c. means the complex conjugate with respect to $z_{k}$. As has been shown in refs. [33] and [34], as soon as the inequality $2(n+1)>$ $\max \left(P_{t}, \Omega-P_{t}\right)$ is satisfied, the projected state coincides with the $P_{t}$ pairs of the particles component.

In the np pairing case, the projector that allows one to obtain the state that has both the good proton and neutron numbers (and hence the good isospin) is then of the form

$$
\hat{\wp}=\prod_{t=n, p} \widehat{\wp}_{t} .
$$

The projected wave function is then

$$
\left|\Psi_{n n^{\prime}}\right\rangle=C_{n n^{\prime}} \hat{\wp}|\Psi\rangle,
$$


where $C_{n n^{\prime}}$ is a normalization factor. That is

$$
\begin{aligned}
\left|\Psi_{n n^{\prime}}\right\rangle= & C_{n n^{\prime}}\left[\sum _ { k = 0 } ^ { n + 1 } \sum _ { k ^ { \prime } = 0 } ^ { n ^ { \prime } + 1 } \xi _ { k } \xi _ { k ^ { \prime } } \left(z_{k}^{-P_{n}} z_{k^{\prime}}^{-P_{p}}\left|\Psi\left(z_{k}, z_{k^{\prime}}\right)\right\rangle\right.\right. \\
& \left.\left.+\bar{z}_{k}^{-P_{n}} z_{k^{\prime}}^{-P_{p}}\left|\Psi\left(\bar{z}_{k}, z_{k^{\prime}}\right)\right\rangle+\text { c.c. }\right)\right]
\end{aligned}
$$

where $\bar{z}_{k}$ is the complex conjugate of $z_{k}$, and

$$
\left|\Psi\left(z_{k}, z_{k^{\prime}}\right)\right\rangle=\prod_{\nu>0}\left|\Psi_{\nu}\left(z_{k}, z_{k^{\prime}}\right)\right\rangle
$$

with

$$
\begin{aligned}
& \left|\Psi_{\nu}\left(z_{k}, z_{k^{\prime}}\right)\right\rangle= \\
& \quad\left[B_{1}^{\nu} z_{k} z_{k^{\prime}} a_{\tilde{\nu} p}^{+} a_{\nu p}^{+} a_{\tilde{\nu} n}^{+} a_{\nu n}^{+}\right. \\
& \quad+B_{2}^{\nu} z_{k^{\prime}} a_{\tilde{\nu} p}^{+} a_{\nu p}^{+}+B_{3}^{\nu} z_{k} a_{\tilde{\nu} n}^{+} a_{\nu n}^{+} \\
& \left.\quad+B_{4}^{\nu} \sqrt{z_{k} z_{k^{\prime}}}\left(a_{\tilde{\nu} p}^{+} a_{\nu n}^{+}+a_{\tilde{\nu} n}^{+} a_{\nu p}^{+}\right)+B_{5}^{\nu}\right]|0\rangle .
\end{aligned}
$$

It appears that the expressions (5) and (17) are formally similar. The effect of the projection in these expressions consists in a renormalization of the $B_{i}^{\nu}$ factors.

The integers $n$ and $n^{\prime}$, respectively, measure the extraction degree of the neutron and proton false components of $|\Psi\rangle$. As soon as the condition

$$
\begin{aligned}
& 2(n+1)>\operatorname{Max}\left(P_{n}, \Omega-P_{n}\right), \\
& 2\left(n^{\prime}+1\right)>\operatorname{Max}\left(P_{p}, \Omega-P_{p}\right),
\end{aligned}
$$

where $2 \Omega$ is the total degeneracy of pairs, is satisfied, the state (15) coincides with the physical component (i.e. with $N$ neutrons and $Z$ protons).

We have thus obtained an explicit expression of the projected wave function. The latter allows one to deduce the expectation value of any physical observable.

The particle-number fluctuations will be measured by

$$
\Delta N_{n n^{\prime}}=\frac{1}{A} \sqrt{\left\langle\Psi_{n n^{\prime}}\left|\hat{N}^{2}\right| \Psi_{n n^{\prime}}\right\rangle-\left\langle\Psi_{n n^{\prime}}|\hat{N}| \Psi_{n n^{\prime}}\right\rangle^{2}} .
$$

\subsection{Energy}

Any operator $\hat{O}$ that conserves the particle-number symmetry satisfies the property

$$
\left\langle\Psi_{n n^{\prime}}|\hat{O}| \Psi_{n n^{\prime}}\right\rangle=4(n+1)\left(n^{\prime}+1\right) C_{n n^{\prime}}\left\langle\Psi_{n n^{\prime}}|\hat{O}| \Psi\right\rangle .
$$

Using (20) the energy of the system is then given by

$$
\begin{aligned}
E_{n n^{\prime}}= & 4(n+1)\left(n^{\prime}+1\right) C_{n n^{\prime}}^{2} \\
& \times\left[\sum _ { k = 0 } ^ { n + 1 } \sum _ { k ^ { \prime } = 0 } ^ { n ^ { \prime } + 1 } \xi _ { k } \xi _ { k ^ { \prime } } \left(z_{k}^{-P_{n}} z_{k^{\prime}}^{-P_{p}} E\left(z_{k}, z_{k^{\prime}}\right)\right.\right. \\
& \left.\left.+\bar{z}_{k}^{-P_{n}} z_{k^{\prime}}^{-P_{p}} E\left(\bar{z}_{k}, z_{k^{\prime}}\right)+\text { c.c. }\right)\right]
\end{aligned}
$$

where

$$
\begin{aligned}
E\left(z_{k}, z_{k^{\prime}}\right) & =\sum_{\nu>0} E_{\nu}\left(z_{k}, z_{k^{\prime}}\right) \prod_{j \neq \nu} A_{j}\left(z_{k}, z_{k^{\prime}}\right) \\
& +\sum_{\nu \neq \mu} E_{\nu \mu}\left(z_{k}, z_{k^{\prime}}\right) \prod_{j \neq \nu \mu} A_{j}\left(z_{k}, z_{k^{\prime}}\right)
\end{aligned}
$$

with

$$
A_{j}\left(z_{k}, z_{k^{\prime}}\right)=\left\langle\Psi_{j}\left(z_{k}, z_{k^{\prime}}\right) \mid \Psi_{j}\right\rangle
$$

$$
\begin{aligned}
& E_{\nu}\left(z_{k}, z_{k^{\prime}}\right)= \\
& z_{k} z_{k^{\prime}}\left(B_{1}^{\nu}\right)^{2}\left(2 \varepsilon_{\nu n}+2 \varepsilon_{\nu p}-\frac{G_{n n}}{4}-\frac{G_{p p}}{4}-\frac{G_{n p}}{2}\right) \\
& +z_{k^{\prime}}\left(B_{2}^{\nu}\right)^{2}\left(2 \varepsilon_{\nu p}-\frac{G_{p p}}{4}\right)+z_{k}\left(B_{3}^{\nu}\right)^{2}\left(2 \varepsilon_{\nu n}-\frac{G_{n n}}{4}\right) \\
& +2 \sqrt{z_{k} z_{k^{\prime}}}\left(B_{4}^{\nu}\right)^{2}\left(\varepsilon_{\nu n}+\varepsilon_{\nu p}-\frac{G_{n p}}{4}\right)
\end{aligned}
$$

and

$$
\begin{aligned}
& E_{\nu \mu}\left(z_{k}, z_{k^{\prime}}\right)= \\
& -\frac{G_{n n}}{4} z_{k}\left(z_{k^{\prime}} B_{1}^{\nu} B_{2}^{\nu}+B_{3}^{\nu} B_{5}^{\nu}\right)\left(z_{k^{\prime}} B_{1}^{\mu} B_{2}^{\mu}+B_{3}^{\mu} B_{5}^{\mu}\right) \\
& -\frac{G_{p p}}{4} z_{k^{\prime}}\left(z_{k} B_{1}^{\nu} B_{3}^{\nu}+B_{2}^{\nu} B_{5}^{\nu}\right)\left(z_{k} B_{1}^{\mu} B_{3}^{\mu}+B_{2}^{\mu} B_{5}^{\mu}\right) \\
& -\frac{G_{n p}}{2} \sqrt{z_{k} z_{k^{\prime}}} B_{4}^{\nu} B_{4}^{\mu}\left(\sqrt{z_{k} z_{k^{\prime}}} B_{1}^{\nu}-B_{5}^{\nu}\right) \\
& \times\left(\sqrt{z_{k} z_{k^{\prime}}} B_{1}^{\mu}-B_{5}^{\mu}\right) .
\end{aligned}
$$

Here again, the expressions (9) (respectively (10)) and (24) (respectively (25)) are formally similar and the particlenumber projection leads to a renormalization of the $B_{i}^{\nu}$ factors.

\section{Numerical results. Discussion}

The previously described method is applied within the framework of a Woods-Saxon deformed mean field with a maximum number of major shells $N_{\max }=12$. The groundstate deformation parameters used in the present work are those of Moller and Nix [35].

\subsection{Pairing strength choice}

The choice of the $G_{n p}$ pairing strength is still an open question. Several propositions have been formulated, let us cite among others:

- Chen and Goswami [36], that have arbitrarily chosen the form

$$
G_{n p}=G_{n n}+\frac{6}{A} \text {. }
$$

- Satula and Wyss [18], for their part, justify their choice,

$$
G_{n p}=\frac{1}{2}\left(G_{n n}+G_{p p}\right)
$$

by assuming, using arguments based on the spin invariance, that, on the $N \simeq Z$ line, $G_{n n(p p)} \simeq G_{n p}$. 
Table 1. Variation of the overlap $\left\langle\Psi \mid \Psi_{n n^{\prime}}\right\rangle$, the particle-number fluctuations $\Delta N_{n n^{\prime}}$ and the projected energy $E_{n n^{\prime}}(\mathrm{MeV})$ as a function of the extraction degrees of the false components $n$ and $n^{\prime}$ for the nucleus ${ }^{36} \mathrm{Ar}$.

\begin{tabular}{|ccccc||ccccc|}
\hline$n$ & $n^{\prime}$ & $\left\langle\Psi \mid \Psi_{n n^{\prime}}\right\rangle(\%)$ & $\Delta N_{n n^{\prime}}$ & $E_{n n^{\prime}}(\mathrm{MeV})$ & $n$ & $n^{\prime}$ & $\left\langle\Psi \mid \Psi_{n n^{\prime}}\right\rangle(\%)$ & $\Delta N_{n n^{\prime}}$ & $E_{n n^{\prime}}(\mathrm{MeV})$ \\
\hline \hline 0 & 0 & 50 & 0.09 & -649.12 & 4 & 0 & 41 & 0.06 & -654.70 \\
0 & 1 & 41 & 0.06 & -653.95 & 4 & 1 & 34 & 0.02 & -659.68 \\
0 & 2 & 41 & 0.06 & -654.36 & 4 & 2 & 34 & 0.00 & -660.09 \\
0 & 3 & 41 & 0.06 & -654.36 & 4 & 3 & 34 & 0.00 & -660.10 \\
0 & 4 & 41 & 0.06 & -654.36 & 4 & 4 & 34 & 0.00 & -660.10 \\
0 & 5 & 41 & 0.06 & -654.36 & 4 & 5 & 34 & 0.00 & -660.10 \\
0 & 6 & 41 & 0.06 & -654.36 & 4 & 6 & 34 & 0.00 & -660.10 \\
0 & 7 & 41 & 0.06 & -654.36 & 4 & 7 & 34 & 0.00 & -660.10 \\
\hline \hline 1 & 0 & 42 & 0.06 & -654.50 & 5 & 0 & 41 & 0.06 & -654.70 \\
1 & 1 & 34 & 0.02 & -659.47 & 5 & 1 & 34 & 0.02 & -659.68 \\
1 & 2 & 34 & 0.01 & -659.89 & 5 & 2 & 34 & 0.00 & -660.09 \\
1 & 3 & 34 & 0.01 & -659.89 & 5 & 3 & 34 & 0.00 & -660.09 \\
1 & 4 & 34 & 0.01 & -659.89 & 5 & 4 & 34 & 0.00 & -660.09 \\
1 & 5 & 34 & 0.01 & -659.89 & 5 & 5 & 34 & 0.00 & -660.10 \\
1 & 6 & 34 & 0.01 & -659.89 & 5 & 6 & 34 & 0.00 & -660.10 \\
1 & 7 & 34 & 0.01 & -659.89 & 5 & 7 & 34 & 0.00 & -660.10 \\
\hline \hline 2 & 0 & 41 & 0.06 & -654.70 & 6 & 0 & 41 & 0.06 & -654.70 \\
2 & 1 & 34 & 0.02 & -659.68 & 6 & 1 & 34 & 0.02 & -659.68 \\
2 & 2 & 34 & 0.00 & -660.09 & 6 & 2 & 34 & 0.00 & -660.09 \\
2 & 3 & 34 & 0.00 & -660.10 & 6 & 3 & 34 & 0.00 & -660.09 \\
2 & 4 & 34 & 0.00 & -660.10 & 6 & 4 & 34 & 0.00 & -660.09 \\
2 & 5 & 34 & 0.00 & -660.10 & 6 & 5 & 34 & 0.00 & -660.09 \\
2 & 6 & 34 & 0.00 & -660.10 & 6 & 6 & 34 & 0.00 & -660.09 \\
2 & 7 & 34 & 0.00 & -660.10 & 6 & 7 & 34 & 0.00 & -660.09 \\
\hline \hline 3 & 0 & 41 & 0.06 & -654.70 & 7 & 0 & 41 & 0.06 & -654.70 \\
3 & 1 & 34 & 0.02 & -659.68 & 7 & 1 & 34 & 0.02 & -659.68 \\
3 & 2 & 34 & 0.00 & -660.09 & 7 & 2 & 34 & 0.00 & -660.09 \\
3 & 3 & 34 & 0.00 & -660.10 & 7 & 3 & 34 & 0.00 & -660.09 \\
3 & 4 & 34 & 0.00 & -660.10 & 7 & 4 & 34 & 0.00 & -660.09 \\
3 & 5 & 34 & 0.00 & -660.10 & 7 & 5 & 34 & 0.00 & -660.09 \\
3 & 6 & 34 & 0.00 & -660.10 & 7 & 6 & 34 & 0.00 & -660.09 \\
3 & 7 & 34 & 0.00 & -660.10 & 7 & 7 & 34 & 0.00 & -660.09 \\
\hline
\end{tabular}

- Chasman [20] sets for the same reason

$$
G_{n p}=\frac{1}{2} G_{n n}=\frac{1}{2} G_{p p} .
$$

- Civitarese et al. propose either the form [1]

$$
G_{n p}=\frac{C(Z)}{A},
$$

where $C(Z)$ is a constant that varies as a function of the considered element, or the form [2]

$$
G_{n p}=C \frac{16}{A+56},
$$

where $C$ is a constant.

The latter expression has also been used by Szpikowski [37].

In the present work, we restrict ourselves to $N=Z$ even-even nuclei of which the "experimental" pairing gap may be deduced from the experimental masses [3] (i.e. with $18 \leq Z \leq 32$ ). The pairing strengths $G_{p p}, G_{n n}$ and $G_{n p}$ are then chosen such as to exactly reproduce the "experimental" values of $\Delta_{p p}, \Delta_{n n}$ and $\Delta_{n p}$. For the study of
$N=Z$ nuclei of higher masses (and of which "experimental" pairing gaps are not known), one should first have to establish by a fit an expression of the pairing strengths from the previously obtained values, and then to extrapolate this expression.

\subsection{Convergence of the method}

We have reported in table 1 , the overlap $\left\langle\Psi \mid \Psi_{n n^{\prime}}\right\rangle$, the particle-number fluctuations $\Delta N_{n n^{\prime}}$ as well as the projected energy $E_{n n^{\prime}}(\mathrm{MeV})$ values as a function of the extraction degrees of the false components $n$ and $n^{\prime}(0 \leq$ $\left.n, n^{\prime} \leq 7\right)$ for the nucleus ${ }^{36} \mathrm{Ar}$ chosen as an example. The values obtained using the BCS theory are respectively $\Delta N_{B C S}=0.09$ and $E_{B C S}=-648.78 \mathrm{MeV}$. From table 1, one can conclude that

i) The convergence of the method is very fast. Indeed, following expression (18), the convergence should be theoretically reached as soon as $n, n^{\prime}>218$, since $\Omega=$ 455. But it clearly appears that the results are stable for $n, n^{\prime}>6$ for all the evaluated quantities. This fact proves the efficiency of the projection method. 
Table 2. Overlap between the BCS and projected wave functions, without inclusion of the np pairing correlations for the proton (first row) and neutron (second row) systems; and with inclusion of the np pairing correlations (third row). Difference between the projected and BCS energies (MeV) without (fourth row) and with (fifth row) inclusion of the np pairing correlations.

\begin{tabular}{|l|ccccccccc|}
\hline Nucleus & ${ }^{32} \mathrm{~S}$ & ${ }^{36} \mathrm{Ar}$ & ${ }^{40} \mathrm{Ca}$ & ${ }^{44} \mathrm{Ti}$ & ${ }^{48} \mathrm{Cr}$ & ${ }^{52} \mathrm{Fe}$ & ${ }^{56} \mathrm{Ni}$ & ${ }^{60} \mathrm{Zn}$ & ${ }^{64} \mathrm{Ge}$ \\
\hline \hline$\left\langle\Psi \mid \Psi_{n}\right\rangle_{p}(\%)$ & 59 & 58 & 62 & 56 & 56 & 58 & 56 & 56 & 55 \\
$\left\langle\Psi \mid \Psi_{n}\right\rangle_{n}(\%)$ & 59 & 59 & 63 & 57 & 57 & 59 & 56 & 57 & 54 \\
$\left\langle\Psi \mid \Psi_{n n^{\prime}}\right\rangle(\%)$ & 36 & 34 & 39 & 34 & 32 & 35 & 32 & 35 & 31 \\
$\delta E(\mathrm{MeV})$ & -4.17 & -4.86 & -7.28 & -4.18 & -4.12 & -4.83 & -4.40 & -3.50 & -4.26 \\
$\delta E^{n p}(\mathrm{MeV})$ & -9.59 & -11.31 & -17.72 & -11.57 & -8.72 & -11.00 & -8.63 & -7.84 & -9.13 \\
\hline
\end{tabular}

ii) The particle-number projection is indispensable since the overlap $\left\langle\Psi \mid \Psi_{n n^{\prime}}\right\rangle$ between the BCS and projected wave function is only $34 \%$ (after convergence). It is thus very far from the theoretical overlap.

iii) The particle-number fluctuations are efficiently eliminated since the quantity $\Delta N$ that was 0.09 with the BCS theory vanishes after projection.

iv) The energy value is significantly reduced with regard to the BCS one (the difference is more than $11 \mathrm{MeV}$ ).

\subsection{Effects of the particle-number fluctuations on the system energy}

In order to evaluate the particle-number fluctuations effect on the system energy, we have calculated the difference between the projected and BCS energies,

$$
\delta E^{n p}=E_{n n^{\prime}}-E_{B C S}^{n p},
$$

for the ground state of the previously cited nuclei. The obtained values are compared in table 2 to those of the same quantity, when only the pairing between like-particles is taken into account, i.e.

$$
\delta E=E_{n}-E_{B C S}
$$

where $E_{n}$ is the energy evaluated using the SBCS particlenumber projection method $[33,34,38]$.

It then appears that the particle-number fluctuations effects are even more important when the np pairing is considered. Indeed, the $\delta E$ value is on average $-4.62 \mathrm{MeV}$, whereas that of $\delta E^{n p}$ is $-10.61 \mathrm{MeV}$.

We have also reported in the same table the overlap between the BCS and projected wave functions without $\left(\left\langle\Psi \mid \Psi_{n}\right\rangle_{p}\right.$ and $\left\langle\Psi \mid \Psi_{n}\right\rangle_{n}$ for the proton and neutron systems, respectively) and with $\left(\left\langle\Psi \mid \Psi_{n n^{\prime}}\right\rangle\right)$ inclusion of the np pairing correlations. Here again, it appears that the particlenumber fluctuations are even more important when the $\mathrm{np}$ correlations are included. Indeed, the average value of $\left\langle\Psi \mid \Psi_{n}\right\rangle_{p}$ or $\left\langle\Psi \mid \Psi_{n}\right\rangle_{n}$ is $57 \%$, whereas that of $\left\langle\Psi \mid \Psi_{n n^{\prime}}\right\rangle$ is only $34 \%$.

These facts show the necessity of both the inclusion of the np pairing correlations and the particle-number projection in $N \simeq Z$ nuclei. It will be the case in the astrophysical context and specially for the study of the rp-process. Indeed, as underlined in the introduction, this study is not only hindered by the uncertainty of the astrophysical conditions but also by the lack of experimental information on the nuclei along this process. The theoretical predictions have thus to be particularly rigorous.

Finally, it is worth noticing that the present particlenumber projection method could be easily generalized to the excited states and hence used for the double-beta decay study.

\section{References}

1. O. Civitarese, M. Reboiro, Phys. Rev. C 56, 1179 (1997).

2. O. Civitarese, M. Reboiro, P. Vogel, Phys. Rev. C 56, 1840 (1997).

3. F. Simkovic, Ch.C. Moustakidis, L. Pacearescu, A. Faessler, Phys. Rev. C 68, 054319 (2003).

4. J. Engel, S. Pittel, M. Stoitsov, P. Vogel, J. Dukelsky, Phys. Rev. C 55, 1781 (1997).

5. A.L. Goodman, Phys. Rev. C 60, 014311 (1999).

6. D.R. Bes, O. Civitarese, E.E. Moqueda, N.N. Scoccola, Phys. Rev. C 61, 024315 (2000).

7. D. Mokhtari, N.H. Allal, M. Fellah, Heavy Ion Phys. 19, 187 (2004).

8. A. Goodman, Adv. Nucl. Phys. 11, 263 (1979).

9. G. Pantis, F. Simkovic, J.D. Vergados, A. Faessler, Phys. Rev. C 53, 695 (1996).

10. J. Dobes, S. Pittel, Phys. Rev. C 57, 688 (1998).

11. O. Civitarese, F. Montani, M. Reboiro, Phys. Rev. C 60, 24305 (1999).

12. A. Bobyk, W.A. Kaminski, P. Zareba, Eur. Phys. J. A 5, 385 (1999).

13. S.G. Frauendorf, J.A. Sheikh, Nucl. Phys. A 645, 509 (1999).

14. D.S. Delion, J. Dukelsky, P. Schuck, E.J.V. de Passos, F. Krmpotic, Phys. Rev. C 62, 44311 (2000).

15. A.A. Raduta, L. Pacearescu, V. Baran, P. Sarriguren, E. Moya de Guerra, Nucl. Phys. A 675, 503 (2000).

16. P. Sarriguren, E. Moya de Guerra, R. Alvarez-Rodriguez, Nucl. Phys. A 716, 230 (2003).

17. N. Paar, T. Niksic, D. Vretenar, P. Ring, Phys. Rev. C 69, 054303 (2004).

18. W. Satula, R. Wyss, Nucl. Phys. A 676, 120 (2000).

19. M. Kyotoku, H.T. Chen, Phys. Rev. C 36, 1144 (1987).

20. R.R. Chasman, Phys. Lett. B 524, 81 (2002).

21. Perlinska, S.G. Rohozinski, J. Dobaczewski, W. Nazarewicz, Phys. Rev. C 69, 014316 (2004).

22. Y. Sun, Eur. Phy. J. A 20, 133 (2004).

23. K. Kaneko, M. Hasegawa, Phys. Rev. C 72, 031302 (2005).

24. A.A. Raduta, O. Haug, F. Simkovic, A. Faessler, J. Phys. G 27, 2429 (2001).

25. J. Suhonen, M. Aunola, Nucl. Phys. A 723, 271 (2003). 
26. H.V. Klapdor-Kleingrothaus, A. Dietz, I.V. Krivosheina, Ch. Dorr, C. Tomei, Phys. Lett. B 578, 54 (2004).

27. R. Arnold et al., Phys. Rev. Lett. 95, 182302 (2005).

28. N. Gupta, H.S. Mani, J. Phys. G 31, 599 (2005).

29. N.H. Allal, M. Fellah, M.R. Oudih, N. Benhamouda, Exotic Nuclei, EXON2004, Proceedings of the International Symposium, Peterhof, 2004, edited by Yu. E. Penionzhkevich, E.A. Cherepanov (World Scientific, Singapore, 2005). 30. R.W. Richardson, N. Sherman, Nucl. Phys. 52, 253 (1964). 31. A. Goswami, Nucl. Phys. 60, 228 (1964).
32. M.R. Oudih, M. Fellah, N.H. Allal, Int. J. Mod. Phys. E 12, 109 (2003).

33. V.N. Fomenko, J. Phys. A 3, 8 (1970).

34. M. Fellah, T.F. Hammann, Nuovo Cimento A 30, 239 (1975).

35. P. Moller, J.R. Nix, W.D. Myers, W.J. Swiatecki, At. Data Nucl. Data Tables 59, 185 (1995).

36. H. Chen, A. Goswami, Phys. Lett. B 24, 257 (1967).

37. S. Szpikowski, Acta Phys. Pol. B 31, 443 (2000).

38. M. Fellah, T.F. Hammann, D.E. Medjadi, Phys. Rev. C 8, 1585 (1973). 